\title{
Wirkung von Salzen auf die Autolyse.
}

\author{
Von
}

Dr. Luigi Preti, Assistent des Instituts.

(Aus dem Institute für spezielle Pathologie innerer Krankheiten der K. Universität Pavia, Professor M. Ascoli.)

(Der Redaktion zugegangen am 11. Mai 1909.)

In einer früher veröffentlichten Arbeit $\left.{ }^{1}\right)$ wurde nachgewiesen, daß Bleiacetat sowie Bleinitrat auf die Leberautolyse, je nach der Menge in der sie angewendet werden, eine fördernde oder hemmende Wirkung ausüben.

Diese Untersuchungen, welche darauf hinzielten, einige Besonderheiten aufzuklären, die aus Untersuchungen ${ }^{2}$ ) über den Stickstoffumsatz von drei mit Blei vergifteten Individuen erhalten waren, verdienten auch auf die Salze anderer Metalle ausgedehnt zu werden, um zu erforschen, welchen Einfluß diese auf einen so komplizierten und wichtigen Prozeß ausüben können, wie es die Leberautolyse ist. Die Ergebnisse dieser Forschungen waren auch deshalb von Bedeutung, weil sie als Anhaltspunkte für einen Vergleich mit der Wirkung dienen konnten, welchen auf denselben Prozeß die gleichen Metalle in der Hydrosolform ausüben (M. Ascoli und G. Izar ${ }^{3}$ ).)

Ich habe die Salze folgender Metalle untersucht: Eisen, Mangan, Gold, Platin, Kobalt, Aluminium, Magnesium, Kalium, Natrium, Kupfer, Strontium, Nickel, Cadmium, Palladium. (Die Versuche mit den Silber- und Quecksilbersalzen werden getrennt ausgeführt werden.)

1) Diese Zeitschrift, Bd. LVIII.

2) Deutsch. Archiv f. klin. Medizin, Bd. XCV.

$\left.{ }^{3}\right)$ Biochemische Zeitschrift, Bd. VI u. VII. 
Den Aufschwemmungen von Kalbsleberbrei setzte ich steigende Mengen der genannten Salze, in Form von äquimolekulären Lösungen, hinzu, und überließ sie 3 Tage der Autolyse, als Kontrollprobe wurde ein Versuch ohne irgend welchen Zusatz ausgeführt. Bei anderen Reihen von Kontrollproben mit Zusatz der Salze wurde die Masse nach der gewöhnlichen Methode sofort zum Gerinnen gebracht. ${ }^{1}$ )

Für jedes Salz wurden die Versuche mit ein und demselben Leberbrei ausgeführt.

\section{Eisensalze.}

Unter den Eisensalzen wurden folgende in bezug auf ihre Wirkung untersucht: Eisenchlorid, Eisensulfat(oxyd), oxalsaures Eisen (oxyd), Eisenchlorür, Eisensulfat (oxydul) (Tab. I-V).

Tabelle I.

\begin{tabular}{|c|c|c|c|c|c|}
\hline $\begin{array}{l}\text { Versuchs- } \\
\text { nummer }\end{array}$ & $\begin{array}{l}\text { Dauer } \\
\text { der } \\
\text { Autolyse } \\
\text { Tage }\end{array}$ & $\begin{array}{l}\text { Destil- } \\
\text { liertes } \\
\text { Wasser } \\
\text { ccm }\end{array}$ & $\begin{array}{l}\text { Eisen- } \\
\text { chlorid- } \\
\text { lösung } \\
\text { ccm }\end{array}$ & $\begin{array}{c}\text { Fe- } \\
\text { Gehalt } \\
\text { g }\end{array}$ & $\begin{array}{l}\text { Nicht koagulierter } \mathrm{N} \\
\text { in } 50 \mathrm{ccm} \text { Filtrat } \\
\text { entsprechend } 1 / 10-\mathrm{n}- \\
\text { Säure } \\
\text { in } \mathrm{ccm}\end{array}$ \\
\hline 1 & 0 & 250 & - & - & 7 \\
\hline 2 & 0 & 248 & $1 / 100-n \quad 2$ & 0,001118 & 7,1 \\
\hline 3 & 0 & 230 & $\begin{array}{l}-20 \\
\end{array}$ & 0,01118 & 7,9 \\
\hline 4 & 0 & 200 & $\gg \quad 50$ & 0,02995 & 8,0 \\
\hline 5 & 0 & 150 & , 100 & 0,0559 & 9,9 \\
\hline 6 & 3 & 250 & 一 & - & 11,1 \\
\hline 7 & 3 & 248 & $1 / 100-n \quad 2$ & 0,001118 & 11,7 \\
\hline 8 & 3 & 230 & $\begin{array}{l}> \\
20\end{array}$ & 0,01188 & 11,9 \\
\hline 9 & 3 & 200 & , 50 & 0,02995 & 13,4 \\
\hline 10 & 3 & 150 & $\gg \quad 100$ & 0,0559 & 16,4 \\
\hline
\end{tabular}

1) Bezüglich der übrigen technischen Einzelheiten (7\% Chloroformzusatz usw.) verweise ich auf meine frühere Arbeit in dieser Zeitschrift, Bd. LVIII. 
Tabelle II.

\begin{tabular}{|c|c|c|c|c|c|}
\hline $\begin{array}{l}\text { Versuchs- } \\
\text { nummer }\end{array}$ & $\begin{array}{l}\text { Dauer } \\
\text { der } \\
\text { Autolyse } \\
\text { Tage }\end{array}$ & $\begin{array}{l}\text { Destil- } \\
\text { liertes } \\
\text { Wasser } \\
\text { ccm }\end{array}$ & $\begin{array}{l}\text { Eisen- } \\
\text { sulfat } \\
\text { (oxyd)- } \\
\text { lösung } \\
\text { ccm }\end{array}$ & $\begin{array}{l}\text { Fe- } \\
\text { Gehalt } \\
\text { g }\end{array}$ & $\begin{array}{l}\text { Nicht koagulierter } N \\
\text { in } 50 \mathrm{ccm} \text { Filtrat } \\
\text { entsprechend } 1 / 10-\mathrm{n}- \\
\text { Säure } \\
\text { in ccm }\end{array}$ \\
\hline 1 & 0 & 250 & - & - & 11,0 \\
\hline 2 & 0 & 248 & $1 / 100-n \quad 2$ & 0,001118 & 11,1 \\
\hline 3 & 0 & 230 & $\bowtie \quad 20$ & 0,01118 & 10,9 \\
\hline 4 & 0 & 200 & $\triangle \quad 50$ & 0,02995 & 13,2 \\
\hline j & 0 & 150 & $\gg \quad 100$ & 0,0559 & 15,1 \\
\hline 6 & 3 & 250 & - & - & 16,5 \\
\hline 7 & 3 & 248 & $1 / 100-n \quad 2$ & 0,001118 & 16,2 \\
\hline 8 & 3 & 230 & 20 & 0,01118 & 16,3 \\
\hline 9 & 3 & 200 & $\rtimes \quad 50$ & 0,02995 & 22,9 \\
\hline 10 & 3 & 150 & $\begin{array}{l}\times \\
\end{array}$ & 0,0559 & 31,8 \\
\hline
\end{tabular}

Tabelle III.

\begin{tabular}{|c|c|c|c|c|c|}
\hline $\begin{array}{c}\text { Versuchs- } \\
\text { nummer }\end{array}$ & $\begin{array}{l}\text { Dauer } \\
\text { der } \\
\text { Autolyse } \\
\text { Tage }\end{array}$ & $\begin{array}{l}\text { Destil- } \\
\text { liertes } \\
\text { Wasser } \\
\text { ccm }\end{array}$ & $\begin{array}{c}\text { Oxalsaure } \\
\text { Eisen } \\
\text { (oxyd)- } \\
\text { lösung } \\
\text { ccm }\end{array}$ & $\begin{array}{c}\text { Fe- } \\
\text { Gehalt } \\
\text { g }\end{array}$ & $\begin{array}{c}\text { Nicht koagulierter } N \\
\text { in } 50 \mathrm{ccm} \text { Filtrat } \\
\text { entsprechend } 1 / 10-\mathrm{n} \\
\text { Saure } \\
\text { in } \mathrm{ccm}\end{array}$ \\
\hline 1 & 0 & 250 & - & - & 9,0 \\
\hline 2 & 0 & 248 & $1 / 100-n \quad 2$ & 0,001118 & 8,8 \\
\hline 3 & 0 & .230 & > 20 & 0,01118 & 9,2 \\
\hline 4 & 0 & 200 & 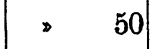 & 0,02995 & 9,0 \\
\hline 5 & 0 & 150 & , 100 & 0,0559 & 9,3 \\
\hline 6 & 3 & 250 & - & - & 13,6 \\
\hline 7 & 3 & 248 & $1 / 100-n \quad 2$ & 0,001118 & 13,5 \\
\hline 8 & 3 & 230 & $\Rightarrow \quad 20$ & 0,01118 & 15,4 \\
\hline 9 & 3 & 200 & $=\quad 50$ & 0,02995 & 18,6 \\
\hline 10 & 3 & 150 & > 100 & 0,0559 & 21,6 \\
\hline
\end{tabular}

Hoppe-Seyler's Zeitschrift f. physiol. Chemie. LX. 
Luigi Preti,

Tabelle IV.

\begin{tabular}{|c|c|c|c|c|c|}
\hline $\begin{array}{l}\text { Versuchs- } \\
\text { nummer }\end{array}$ & $\begin{array}{c}\text { Dauer } \\
\text { der } \\
\text { Autolyse } \\
\text { Tage }\end{array}$ & $\begin{array}{l}\text { Destil- } \\
\text { liertes } \\
\text { Wasser } \\
\text { ccm }\end{array}$ & $\begin{array}{l}\text { Eisen- } \\
\text { chlorür- } \\
\text { lösung } \\
\text { ccm }\end{array}$ & $\begin{array}{c}\text { Fe- } \\
\text { Gehalt } \\
\text { g }\end{array}$ & $\begin{array}{l}\text { Nicht koagulierter } N \\
\text { in } 50 \mathrm{ccm} \text { Filtrat } \\
\text { entsprechend } 1 / 10-\mathrm{n}- \\
\text { Säure } \\
\text { in ccm }\end{array}$ \\
\hline 1 & 0 & 250 & - & - & 9,7 \\
\hline 2 & 0 & 248 & $1 / 100-n \quad 2$ & 0,001118 & 9,8 \\
\hline 3 & 0 & 230 & $\triangle \quad 20$ & 0,01118 & 9,7 \\
\hline 4 & 0 & 200 & $\triangle \quad 50$ & 0,02995 & 10,0 \\
\hline 5 & 0 & 150 & $\gg \quad 100$ & 0,0559 & 10,2 \\
\hline 6 & 3 & 250 & - & - & 14,5 \\
\hline 7 & 3 & 248 & $1 / 100-n \quad 2$ & 0,001118 & 14,5 \\
\hline 8 & 3 & 230 & $\gg \quad 20$ & 0,01118 & 13,8 \\
\hline 9 & 3 & 200 & $\gg \quad 50$ & 0,02995 & 15,0 \\
\hline 10 & 3 & 150 & $" \quad 100$ & 0,0559 & 16,3 \\
\hline
\end{tabular}

Tabelle V.

\begin{tabular}{|c|c|c|c|c|c|}
\hline $\begin{array}{l}\text { Versuchs- } \\
\text { nummer }\end{array}$ & $\begin{array}{l}\text { Dauer } \\
\text { der } \\
\text { Autolyse } \\
\text { Tage }\end{array}$ & $\begin{array}{l}\text { Destil- } \\
\text { liertes } \\
\text { Wasser } \\
\text { ccm }\end{array}$ & $\begin{array}{c}\text { Eisen- } \\
\text { sulfat } \\
\text { (oxydul)- } \\
\text { lösung } \\
\text { ccm }\end{array}$ & $\begin{array}{c}\text { Fe- } \\
\text { Gehalt } \\
\text { g }\end{array}$ & $\begin{array}{l}\text { Nicht koagulierter } N \\
\text { in } 50 \mathrm{ccm} \text { Filtrat } \\
\text { entsprechend } 1 / 10-\mathrm{n}- \\
\text { Säure } \\
\text { in ccm }\end{array}$ \\
\hline 1 & 0 & 250 & - & - & 10,5 \\
\hline 2 & 0 & 248 & $1 / 100-n \quad 2$ & 0,001118 & 10,4 \\
\hline 3 & 0 & 230 & 》 20 & 0,01118 & 10,6 \\
\hline 4 & 0 & 200 & $\triangle \quad 50$ & 0,02995 & 10,7 \\
\hline 5 & 0 & 150 & $>\quad 100$ & 0,0559 & 10,7 \\
\hline 6 & 3 & 250 & - & - & 13,9 \\
\hline 7 & 3 & 248 & $1 / 100-n \quad 2$ & 0,001118 & 13,5 \\
\hline 8 & 3 & 230 & $» \quad 20$ & 0,01118 & 13,9 \\
\hline 9 & 3 & 200 & $\searrow \quad 50$ & 0,02995 & 15,5 \\
\hline 10 & 3 & 150 & \100 & 0,0559 & 17,0 \\
\hline
\end{tabular}


Aus den Tabellen I-V geht hervor, daß die nach vollendeter Autolyse nachweisbare Menge des nicht koagulierbaren Stickstoffs bei den Versuchen mit Zusatz dieser Salze größer war als bei den Kontrollproben ohne Zusatz derselben.

Die Zunahme beobachtet man, wenn die Menge des Salzes $2 \mathrm{ccm}$ einer $1 / 100$-Normallösung überschreitet, und geht parallel jener der zugesetzten Salzmenge vor sich.

Es sei jedoch hervorgehoben, daß bei den Proben mit Zusatz von Eisenchlorid resp. Sulfat diese Zunahme auch vor der Autolyse beobachtet wird.

2. Mangansalze.

Es wurden folgende untersucht: Chlorid, Sulfat, Acetat und milchsaures (Tabelle VI-IX).

Tabelle VI.

\begin{tabular}{|c|c|c|c|c|c|}
\hline $\begin{array}{c}\text { Versuchs- } \\
\text { nummer }\end{array}$ & $\left|\begin{array}{c}\text { Dauer der } \\
\text { Autolyse } \\
\text { Tage }\end{array}\right|$ & $\begin{array}{l}\text { Destil- } \\
\text { liertes } \\
\text { Wasser } \\
\text { ccm }\end{array}$ & $\begin{array}{l}\text { Mangan- } \\
\text { chlorür- } \\
\text { lösung } \\
\mathrm{ccm}\end{array}$ & $\begin{array}{c}\text { Mn- } \\
\text { Gehalt } \\
\mathbf{g}\end{array}$ & $\begin{array}{c}\text { Nicht koagulierter } N \\
\text { in } 50 \text { ccm Filtrat } \\
\text { entspr. } 1 / 10-n-S a ̈ u r e \\
\text { in ccm }\end{array}$ \\
\hline 1 & 0 & 250 & 一 & 一 & 9,4 \\
\hline 2 & 0 & 230 & $1 / 100-n \quad 20$ & 0,0110 & 9,4 \\
\hline 3 & 0 & 200 & $\rtimes \quad 50$ & 0,0275 & 9,4 \\
\hline 4 & 0 & 150 & $\Rightarrow \quad 100$ & 0,055 & 9,6 \\
\hline 5 & 0 & 230 & $1 / 10-n \quad 20$ & 0,1100 & 9,9 \\
\hline 6 & 0 & 225 & $\gg \quad 25$ & 0,1375 & 9,9 \\
\hline 7 & 0 & 200 & . 50 & 0,2750 & 11,2 \\
\hline 8 & 0 & 150 & $\triangleright \quad 100$ & 0,5500 & 12,3 \\
\hline 9 & 3 & 250 & - & - & 16,9 \\
\hline 10 & 3 & 230 & $1 / 100-n \quad 20$ & 0,0110 & 16,6 \\
\hline 11 & 3 & 200 & $\triangle \quad 50$ & 0,0275 & 16,5 \\
\hline 12 & 3 & 150 & $\gg \quad 100$ & 0,0550 & 16,1 \\
\hline 13 & 3 & 230 & $1 / 10-n \quad 20$ & 0,1100 & 20,2 \\
\hline 14 & 3 & 225 & $\triangleright \quad 25$ & 0,1375 & 21,7 \\
\hline 15 & 3 & 200 & $\gg \quad 50$ & 0,2750 & 22,5 \\
\hline 16 & 3 & 150 & $>\quad 100$ & 0,5500 & 26,4 \\
\hline
\end{tabular}


Tabelle VII.

\begin{tabular}{|c|c|c|c|c|c|}
\hline $\begin{array}{l}\text { Versuchs- } \\
\text { nummer }\end{array}$ & $\begin{array}{c}\text { Dauer der } \\
\text { Autolyse } \\
\text { Tage }\end{array}$ & $\begin{array}{l}\text { Destil- } \\
\text { liertes } \\
\text { Wasser } \\
\text { ccm }\end{array}$ & \begin{tabular}{|c|} 
Mangan- \\
sulfat- \\
lösung \\
ccm
\end{tabular} & $\begin{array}{l}\text { Mn- } \\
\text { Gehalt } \\
\text { g }\end{array}$ & $\begin{array}{c}\text { Nicht koagulierter } N \\
\text { in } 50 \mathrm{ccm} \text { Filtrat } \\
\text { entspr. } 1 / 10-n-\text { Säure } \\
\text { in } \mathrm{ccm}\end{array}$ \\
\hline 1 & 0 & 250 & - & - & 10,5 \\
\hline 2 & 0 & 230 & $1 / 100-n \quad 20$ & 0,0110 & 10,8 \\
\hline 3 & 0 & 200 & $\triangleright \quad 50$ & 0,0275 & 10,8 \\
\hline 4 & 0 & 150 & $\searrow \quad 100$ & 0,0550 & 10,6 \\
\hline 5 & 0 & 230 & $1 / 10-n \quad 20$ & 0,1100 & 10,8 \\
\hline 6 & 0 & 225 & $\triangleright$ & 0,1375 & 10,7 \\
\hline 7 & 0 & 200 & 50 & 0,2750 & 11,9 \\
\hline 8 & 0 & 150 & $\triangleright \quad 100$ & 0,5500 & 12,0 \\
\hline 9 & 3 & 250 & - & - & 12,6 \\
\hline 10 & 3 & 230 & $1 / 100-n \quad 20$ & 0,0110 & 12,1 \\
\hline 11 & 3 & 200 & 50 & 0,0275 & 12,8 \\
\hline 12 & 3 & 150 & $\bowtie \quad 100$ & 0,0550 & 13,2 \\
\hline 13 & 3 & 230 & $1 / 10-n \quad 20$ & 0,1100 & 14,9 \\
\hline 14 & 3 & 225 & » & 0,1375 & 16,9 \\
\hline 15 & 3 & 200 & 50 & 0,2750 & 17,7 \\
\hline 16 & 3 & 150 & $\triangleright \quad 100$ & 0,5500 & 17,4 \\
\hline
\end{tabular}

Tabelle VIII.

\begin{tabular}{|c|c|c|c|c|c|}
\hline $\begin{array}{l}\text { Versuchs- } \\
\text { nummer }\end{array}$ & $\begin{array}{c}\text { Dauer der } \\
\text { Autolyse } \\
\text { Tage }\end{array}$ & $\begin{array}{c}\text { Destil- } \\
\text { liertes } \\
\text { Wasser } \\
\text { ccm }\end{array}$ & \begin{tabular}{|} 
Essigsaure \\
Mangan- \\
lösung \\
$\mathrm{ccm}$
\end{tabular} & $\begin{array}{c}\text { Mn- } \\
\text { Gehalt } \\
\mathrm{g}\end{array}$ & $\begin{array}{c}\text { Nicht koagulierter } N \\
\text { in } 50 \mathrm{ccm} \text { Filtrat } \\
\text { entspr. 1/10-n-Säure } \\
\text { in ccm }\end{array}$ \\
\hline 1 & 0 & 250 & - & - & 9,9 \\
\hline 2 & 0 & 230 & $1 / 100-n \quad 20$ & 0,0110 & 9,6 \\
\hline 3 & 0 & 200 & $\gg \quad 50$ & 0,0275 & 10,0 \\
\hline 4 & 0 & 150 & $\triangleright \quad 100$ & 0,0550 & 10,2 \\
\hline 5 & 0 & 230 & $1 / 10-n \quad 20$ & 0,1100 & 10,5 \\
\hline 6 & 0 & 225 & 25 & 0,1375 & 10,2 \\
\hline 7 & 0 & 200 & 50 & 0,2750 & 10,4 \\
\hline 8 & 0 & 150 & » 100 & 0,5500 & 10,5 \\
\hline$y$ & 3 & 250 & - & - & 15,4 \\
\hline 10 & 3 & 230 & $1 / 100-n \quad 20$ & 0,0110 & 15,4 \\
\hline 11 & 3 & 200 & $» \quad 50$ & 0,0275 & 15,4 \\
\hline 12 & 3 & 150 & 100 & 0,0550 & 16,3 \\
\hline 13 & 3 & 230 & $1 / 10-n \quad 20$ & 0,1100 & 18,5 \\
\hline 14 & 3 & 225 & 25 & 0,1375 & 19,3 \\
\hline 15 & 3 & 200 & 50 & 0,2750 & 18,6 \\
\hline 16 & 3 & 150 & - 100 & 0,5500 & 17,3 \\
\hline
\end{tabular}


Tabelle IX.

\begin{tabular}{|c|c|c|c|c|c|}
\hline $\begin{array}{l}\text { Versuchs- } \\
\text { nummer }\end{array}$ & $\left|\begin{array}{c}\text { Dauer der } \\
\text { Autolyse } \\
\text { Tage }\end{array}\right|$ & $\begin{array}{l}\text { Destil- } \\
\text { liertes } \\
\text { Wasser } \\
\text { ccm }\end{array}$ & $\left|\begin{array}{c}\text { Milchsaure } \\
\text { Mangan- } \\
\text { lösung } \\
\text { ccm }\end{array}\right|$ & $\begin{array}{l}\text { Mn- } \\
\text { Gehalt } \\
\text { g }\end{array}$ & $\begin{array}{c}\text { Nicht koagulierter N } \\
\text { in } 50 \mathrm{ccm} \text { Filtrat } \\
\text { entspr. } 1 / 10-\mathrm{n}-\text { Säure } \\
\text { in ccm }\end{array}$ \\
\hline 1 & 0 & 250 & - & - & 10,0 \\
\hline 2 & 0 & 230 & $1 / 100-n \quad 20$ & 0,0110 & 9,4 \\
\hline 3 & 0 & 200 & $\searrow \quad 50$ & 0,0275 & 9,8 \\
\hline 4 & 0 & 150 & $\triangleright \quad 100$ & 0,0550 & 10,0 \\
\hline 5 & 0 & 230 & $1 / 10-n \quad 20$ & 0,1100 & 9,6 \\
\hline 6 & 0 & 225 & $\triangleright \quad 25$ & 0,1375 & 10,4 \\
\hline 7 & 3 & 250 & 一 & - & 16,0 \\
\hline 8 & 3 & 248 & $1 / 100-n \quad 2$ & 0,0011 & 16,3 \\
\hline 9 & 3 & 230 &  & 0,0111 & 16,0 \\
\hline 10 & 3 & 200 & $\rtimes \quad 50$ & 0,0275 & 17,6 \\
\hline 11 & 3 & 150 & $\gg \quad 100$ & 0,0550 & 19,2 \\
\hline 12 & 3 & 230 & $1 / 10-n \quad 20$ & 0,1110 & 22,6 \\
\hline 13 & 3 & 225 & $\gg \quad 25$ & 0,1375 & 22,4 \\
\hline 14 & 3 & 200 & $\gg \quad 50$ & 0,2750 & 24,4 \\
\hline 15 & 3 & 150 & . 100 & 0,5500 & 26,8 \\
\hline
\end{tabular}

Aus den Resultaten meiner Versuche geht hervor, daß die nach stattgefundener Autolyse nachweisbare Menge des nicht koagulierbaren Stickstoffs bei den Proben mit Zusatz der Mangansalze zugenommen hat, und daß diese Zunahme parallel mit dem Steigen der Menge des zugesetzten Salzes schreitet.

Es muß jedoch hervorgehoben werden, daß große Salzmengen auch bei den Proben, bei welchen die N-Bestimmung vor der Autolyse ausgeführt wurde, eine Zunahme des Stickstoffs bewirkte.

Bezüglich der Wirkung des Manganacetats sehen wir, daß, wenn die Menge des zugesetzten Salzes eine gewisse Grenze erreicht hat, die Zunahme aufhört und einer Abnahme den Platz räumt. 
3. Goldchlorid.

Tabelle X.

\begin{tabular}{|c|c|c|c|c|c|}
\hline $\begin{array}{l}\text { Versuchs- } \\
\text { nummer }\end{array}$ & $\begin{array}{l}\text { Dauer } \\
\text { der } \\
\text { Autolyse } \\
\text { Tage }\end{array}$ & $\begin{array}{l}\text { Destil- } \\
\text { liertes } \\
\text { Wasser } \\
\text { ccm }\end{array}$ & $\begin{array}{l}\text { Gold- } \\
\text { chlorid- } \\
\text { lösung } \\
\text { ccm }\end{array}$ & $\begin{array}{c}\text { Au- } \\
\text { Gehalt } \\
\text { g }\end{array}$ & $\begin{array}{l}\text { Nicht koagulierter } \mathrm{N} \\
\text { in } 50 \mathrm{ccm} \text { Filtrat } \\
\text { entsprechend } 1 / 10-\mathrm{n}- \\
\text { Säure } \\
\text { in ccm }\end{array}$ \\
\hline 1 & 0 & 250 & - & - & 9,9 \\
\hline 2 & 0 & 248 & $1 / 100-n \quad 2$ & 0,00295 & 9,9 \\
\hline 3 & 0 & 240 & » 10 & 0,01972 & 10,0 \\
\hline 4 & 0 & 200 & $\triangle \quad 50$ & 0,0986 & 12,9 \\
\hline 5 & 0 & 240 & $1 / 10-n \quad 10$ & 0,1972 & 14,7 \\
\hline 6 & 3 & 250 & - & - & 15,4 \\
\hline 7 & 3 & 248 & $1 / 100-n \quad 2$ & 0,00295 & 16,2 \\
\hline 8 & 3 & 240 & $\gg \quad 10$ & 0,01972 & 17,4 \\
\hline 9 & 3 & 200 & × 50 & 0,0986 & 20,2 \\
\hline 10 & 3 & 240 & $1 / 10-n \quad 10$ & 0,1972 & $33, \tilde{5}$ \\
\hline
\end{tabular}

Dadurch, daß man dem Leberbrei Goldchlorid zusetzt, bewirkt man bei den nicht der Autolyse unterworfenen Proben eine geringe Zunahme des nicht koagulierbaren Stickstoffs; bei den Proben mit Autolyse ist diese Zunahme bedeutend stärker. Im ersten Fall beobachtet man erst bei Zusatz von $50 \mathrm{ccm}$ einer $1 / 100-\mathrm{N}$-Lösung eine merkbare Zunahme; im zweiten genügen $2 \mathrm{ccm}$ derselben Lösung.

\section{Platinchlorid.}

Die Menge Platinchlorid welche dem Leberbrei zugesetzt wurde, schwankt zwischen 1 und $40 \mathrm{ccm}$ von einer $1 / 100-\mathrm{N}-$ Lösung.

Bei den Proben mit sofortiger Gerinnung nahm die Menge des nicht koagulierbaren Stickstoffs entsprechend dem $\mathrm{Zu}$ nehmen der zugesetzten Menge von Platinchlorid ab.

Dagegen findet bei den der Autolyse überlassenen Proben das Gegenteil statt: kleine Mengen (1-3 ccm einer ${ }^{1 / 100-N-}$ Lösung) bewirken eine Zunahme des nicht koagulierbaren Stick- 
stoffs, $5 \mathrm{ccm}$ eine geringe Abnahme, während diese wieder einer Zunahme den Platz räumt, wenn man die Menge des zugesetzten Salzes (10-15 ccm derselben Lösung) weiter erhöht; der Zusatz noch größerer Mengen bewirkt wieder eine progressive Abnahme.

Tabelle XI.

\begin{tabular}{|c|c|c|c|c|c|}
\hline $\begin{array}{c}\text { Versuchs- } \\
\text { nummer }\end{array}$ & $\begin{array}{l}\text { Dauer } \\
\text { der } \\
\text { Autolyse } \\
\text { Tage }\end{array}$ & $\begin{array}{l}\text { Destil- } \\
\text { liertes } \\
\text { Wasser } \\
\text { ccm }\end{array}$ & $\begin{array}{l}\text { Platin- } \\
\text { chlorür- } \\
\text { lösung } \\
\mathrm{ccm}\end{array}$ & $\begin{array}{l}\text { Pt- } \\
\text { Gehalt } \\
\text { g }\end{array}$ & $\begin{array}{l}\text { Nicht koagulierter } N \\
\text { in } 50 \mathrm{ccm} \text { Filtrat } \\
\text { entsprechend } 1 / 10-\mathrm{n}- \\
\text { Säure } \\
\text { in ccm }\end{array}$ \\
\hline 1 & 0 & 250 & - & - & 9,8 \\
\hline 2 & 0 & 249 & $.1 / 100-n \quad 1$ & 0,0019 & 9,5 \\
\hline 3 & 0 & 247 & - 3 & 0,0057 & 9,5 \\
\hline 4 & 0 & 245 & $\triangleright \quad 5$ & 0,0095 & 9,4 \\
\hline 5 & 0 & 240 & > 10 & 0,0194 & 9,3 \\
\hline 6 & 0 & 235 & > 15 & 0,0289 & 9,0 \\
\hline 7 & 0 & 230 & $\triangle \quad 20$ & 0,0388 & 8,8 \\
\hline 8 & 0 & 225 & $\triangleright \quad 25$ & 0,0483 & 7,4 \\
\hline 9 & 0 & 210 & $\triangle \quad 40$ & 0,0776 & 6,4 \\
\hline 10 & 3 & 250 & - & - & 16,6 \\
\hline 11 & 3 & 249 & $1 / 100-n \quad 1$ & 0,0019 & 18,6 \\
\hline 12 & 3 & 247 & $\triangleright$ & 0,0057 & 17,9 \\
\hline 13 & 3 & 245 & $\triangleright$ & 0,0095 & 15,8 \\
\hline 14 & 3 & 240 & $\gg \quad 10$ & 0,0194 & 18,1 \\
\hline 15 & 3 & 235 & > 15 & 0,0289 & 18,2 \\
\hline 16 & 3 & 230 & 20 & 0,0388 & 15,1 \\
\hline 17 & 3 & 225 & 25 & 0,0483 & 14,5 \\
\hline 18 & 3 & 210 & 40 & 0,0776 & 13,7 \\
\hline
\end{tabular}

Diese letzte Erscheinung ist aber jedenfalls zum Teil darauf zurückzuführen, daß das Platinchlorid, selbst, wenn es in großen Mengen vorhanden ist, eine Verminderung des nicht koagulierbaren $\mathrm{N}$ hervorruft. 


\section{Aluminiumsalze.}

Es wurden untersucht: das Chlorid und Sulfat (Tab.XII-XIII).

$100 \mathrm{ccm}$ einer $\left.1\right|_{100}-\mathrm{N}-L_{0}$ ösung bewirken, sofort nachdem sie zugesetzt worden sind, eine Zunahme des nicht koagulierbaren $\mathrm{N}$, geringere Mengen lassen diesen unverändert.

Bei Zusatz letzterer Mengen beobachtet man eine progressive Zunahme des Stickstoffs nach vollendeter Autolyse im Vergleich zur Kontrollprobe.

Tabelle XII.

\begin{tabular}{|c|c|c|c|c|c|}
\hline $\begin{array}{l}\text { Versuchs- } \\
\text { nummer }\end{array}$ & $\begin{array}{c}\text { Dauer } \\
\text { der } \\
\text { Autolyse } \\
\text { Tage }\end{array}$ & $\begin{array}{l}\text { Destil- } \\
\text { liertes } \\
\text { Wasser } \\
\text { ccm }\end{array}$ & $\begin{array}{l}\text { Alu- } \\
\text { minium- } \\
\text { chlorid- } \\
\text { lösung } \\
\text { ccm }\end{array}$ & $\begin{array}{l}\text { Al- } \\
\text { Gehalt } \\
\text { g }\end{array}$ & $\begin{array}{c}\text { Nicht koagulierter } \mathrm{N} \\
\text { in } 50 \mathrm{ccm} \text { Filtrat } \\
\text { entsprechend } 1 / 10-\mathrm{n} \text { - } \\
\text { Säure } \\
\text { in ccm }\end{array}$ \\
\hline 1 & 0 & 250 & - & - & 10,9 \\
\hline 2 & 0 & 240 & $1 / 100-n \quad 10$ & 0,00271 & 10,9 \\
\hline 3 & 0 & 230 & $\triangleright \quad 20$ & 0,00542 & 11,0 \\
\hline 4 & 0 & 200 & » 50 & 0,0135 & 11,8 \\
\hline 5 & 0 & 150 & $\times 100$ & 0,027 & 12,8 \\
\hline 6 & 3 & 250 & - & - & 14,9 \\
\hline 7 & 3 & 240 & $1 / 100-n \quad 10$ & 0,00271 & 16,1 \\
\hline 8 & 3 & 230 & » 20 & 0,00542 & 17,2 \\
\hline 9 & 3 & 200 & » 50 & 0,0135 & 20,1 \\
\hline 10 & 3 & 150 & $\triangleright 100$ & 0,027 & 21,6 \\
\hline
\end{tabular}

6. Kobaltsalze.

Ich untersuchte das Kobaltchlorid und -nitrat. Beide bewirken eine progressive Zunahme des $\mathrm{N}$ nach stattgefundener Autolyse.

Bei dem Chlorid wurde die Zunahme nur bis zu einer gewissen Grenze beobachtet; wenn diese überschritten wurde, trat an Stelle der Zunahme eine Abnahme der Menge des nicht koagulierbaren $\mathrm{N}$ ein. 
Tabelle XIII.

\begin{tabular}{|c|c|c|c|c|c|}
\hline $\begin{array}{l}\text { Versuchs- } \\
\text { nummer }\end{array}$ & $\begin{array}{l}\text { Dauer } \\
\text { der } \\
\text { Autolyse } \\
\text { Tage }\end{array}$ & $\begin{array}{l}\text { Destil- } \\
\text { liertes } \\
\text { Wasser } \\
\text { ccm }\end{array}$ & $\begin{array}{l}\text { Alu- } \\
\text { minium- } \\
\text { sulfat- } \\
\text { lösung } \\
\text { ccm }\end{array}$ & $\begin{array}{c}\text { Al- } \\
\text { Gehalt } \\
\text { g }\end{array}$ & $\begin{array}{c}\text { Nicht koagulierter } \mathrm{N} \\
\text { in } 50 \text { ccm Filtrat } \\
\text { entsprechend }\left.{ }^{1}\right|_{10-n-}- \\
\text { Säure } \\
\text { in ccm }\end{array}$ \\
\hline 1 & 0 & 250 & - & - & 11,4 \\
\hline 2 & 0 & 240 & $1 / 100-n \quad 10$ & 0,0027 & 11,2 \\
\hline 3 & 0 & 230 & > 20 & 0,0054 & 11,5 \\
\hline 4 & 0 & 200 & $\gg \quad 50$ & 0,0135 & 11,7 \\
\hline 5 & 0 & 150 & $\triangleright 100$ & 0,027 & 12,0 \\
\hline 6 & 3 & 250 & - & - & 17,7 \\
\hline 7 & 3 & 240 & $1 / 100-n \quad 10$ & 0,0027 & 22,4 \\
\hline 8 & 3 & 230 & $" \quad 20$ & 0,0054 & 29,3 \\
\hline 9 & 3 & 200 & » 50 & 0,0135 & 38,7 \\
\hline 10 & 3 & 150 & $\gg 100$ & 0,027 & 37,0 \\
\hline
\end{tabular}

Tabelle XIV.

\begin{tabular}{|c|c|c|c|c|c|}
\hline $\begin{array}{c}\text { Versuchs- } \\
\text { nummer }\end{array}$ & $\begin{array}{l}\text { Dauer } \\
\text { der } \\
\text { Autolyse } \\
\text { Tage }\end{array}$ & $\begin{array}{l}\text { Destil- } \\
\text { liertes } \\
\text { Wasser } \\
\text { ccm }\end{array}$ & $\begin{array}{l}\text { Kobalt- } \\
\text { chlorür- } \\
\text { lösung } \\
\text { ccm }\end{array}$ & $\begin{array}{c}\text { Co- } \\
\text { Gehalt } \\
\text { g }\end{array}$ & $\begin{array}{l}\text { Nicht koagulierter } N \\
\text { in } 50 \mathrm{ccm} \text { Filtrat } \\
\text { entsprechend } 1 / 10-n- \\
\text { Säure } \\
\text { in ccm }\end{array}$ \\
\hline 1 & 0 & 250 & - & - & 10,3 \\
\hline 2 & 0 & 240 & $1 / 100-n \quad 10$ & 0,0059 & 10,2 \\
\hline 3 & 0 & 230 & , 20 & 0,0118 & 10,4 \\
\hline 4 & 0 & 200 & $\triangleright \quad 50$ & 0,0295 & 10,0 \\
\hline 5 & 0 & 150 & 》 100 & 0,059 & 9,8 \\
\hline 6 & 3 & 250 & - & - & 21,7 \\
\hline 7 & 3 & 248 & $1 / 100-n \quad 2$ & 0,00118 & 22,4 \\
\hline 8 & 3 & 245 & $>\quad 5$ & 0,00295 & 22,8 \\
\hline 9 & 3 & 240 & > 10 & 0,0059 & 23,1 \\
\hline 10 & 3 & 230 & " 20 & 0,0118 & 22,3 \\
\hline 11 & 3 & 200 & > 50 & 0,0295 & 21,6 \\
\hline 12 & 3 & 150 & . 100 & 0,059 & 20,7 \\
\hline
\end{tabular}


Tabelle XV.

\begin{tabular}{|c|c|c|c|c|c|}
\hline $\begin{array}{l}\text { Versuchs- } \\
\text { nummer }\end{array}$ & $\begin{array}{l}\text { Dauer } \\
\text { der } \\
\text { Autolyse } \\
\text { Tage }\end{array}$ & $\begin{array}{l}\text { Destil- } \\
\text { liertes } \\
\text { Wasser } \\
\text { ccm }\end{array}$ & $\begin{array}{l}\text { Kobalt- } \\
\text { nitrat- } \\
\text { lösung } \\
\text { ccm }\end{array}$ & $\begin{array}{c}\text { Co- } \\
\text { Gehalt } \\
\text { g }\end{array}$ & $\begin{array}{l}\text { Nicht koagulierter } N \\
\text { in } 50 \text { ccm Filtrat } \\
\text { entsprechend } 1 / 10-n- \\
\text { Säure } \\
\text { in ccm }\end{array}$ \\
\hline 1 & 0 & 250 & - & - & 11,4 \\
\hline 2 & 0 & 240 & $1 / 100-n \quad 10$ & 0,0059 & 11,3 \\
\hline 3 & 0 & 230 & » 20 & 0,0118 & 11,5 \\
\hline 4 & 0 & 200 & " 50 & 0,0295 & 11,7 \\
\hline 5 & 0 & 150 & $\gg 100$ & 0,059 & 12,1 \\
\hline 6 & 3 & 250 & - & - & 18,5 \\
\hline 7 & 3 & 248 & $1 / 100-n \quad 2$ & 0,00118 & 20,6 \\
\hline 8 & 3 & 245 & $»$ & 0,00295 & 20,7 \\
\hline 9 & 3 & 240 & $\gg \quad 10$ & 0,0059 & 20,3 \\
\hline 10 & 3 & 230 & $\gg \quad 20$ & 0,0118 & 22,0 \\
\hline 11 & 3 & 200 & $\gg \quad 50$ & 0,0295 & 22,1 \\
\hline 12 & 3 & 150 & $\triangle 100$ & 0,059 & 22,0 \\
\hline
\end{tabular}

7. Kupfer- und Natriumsalze.

Tabelle XVI.

\begin{tabular}{|c|c|c|c|c|c|}
\hline $\begin{array}{l}\text { Versuchs- } \\
\text { nummer }\end{array}$ & $\begin{array}{l}\text { Dauer } \\
\text { der } \\
\text { Autolyse } \\
\text { Tage }\end{array}$ & $\begin{array}{l}\text { Destil- } \\
\text { liertes } \\
\text { Wasser } \\
\text { ccm }\end{array}$ & $\begin{array}{l}\text { Kupfer- } \\
\text { sulfat- } \\
\text { lösung } \\
\text { ccm }\end{array}$ & $\begin{array}{l}\text { Cu- } \\
\text { Gehalt } \\
\text { g }\end{array}$ & $\begin{array}{l}\text { Nicht koagulierter } N \\
\text { in } 50 \mathrm{ccm} \text { Filtrat } \\
\text { entsprechend } 1 / 10-\mathrm{n}- \\
\text { Säure } \\
\text { in ccm }\end{array}$ \\
\hline 1 & 0 & 250 & - & - & 9,3 \\
\hline 2 & 0 & 225 & $1 / 100-n \quad 25$ & 0,01575 & 10,4 \\
\hline 3 & 0 & 200 & $\gg \quad 50$ & 0,0315 & 9,1 \\
\hline 4 & 0 & 150 & $\gg \quad 100$ & 0,063 & 9,2 \\
\hline 5 & 3 & 250 & - & - & 13,2 \\
\hline 6 & 3 & 245 & $1 / 100-n \quad 5$ & 0,00315 & 13,4 \\
\hline 7 & 3 & 240 & $\triangle \quad 10$ & 0,0063 & 13,7 \\
\hline 8 & 3 & 225 & 25 & 0,01575 & 11,9 \\
\hline 9 & 3 & 200 & $\supset \quad 50$ & 0,0315 & 10,2 \\
\hline 10 & 3 & 150 & $\triangleright \quad 100$ & 0,063 & 9,0 \\
\hline
\end{tabular}


Wirkung von Salzen auf die Autolyse.

Tabelle XVII.

\begin{tabular}{|c|c|c|c|c|c|}
\hline $\begin{array}{l}\text { Versuchs- } \\
\text { nummer }\end{array}$ & $\begin{array}{l}\text { Dauer } \\
\text { der } \\
\text { Autolyse } \\
\text { Tage }\end{array}$ & $\begin{array}{l}\text { Destil- } \\
\text { liertes } \\
\text { Wasser } \\
\text { ccm }\end{array}$ & $\begin{array}{c}\text { Natrium- } \\
\text { sulfat- } \\
\text { lösung } \\
\text { ccm }\end{array}$ & $\begin{array}{c}\text { Na- } \\
\text { Gehalt } \\
\mathrm{g}\end{array}$ & $\begin{array}{l}\text { Nicht koagulierter N } \\
\text { in } 50 \mathrm{ccm} \text { Filtrat } \\
\text { entsprechend } 1 / 10-n- \\
\text { Säure } \\
\text { in ccm }\end{array}$ \\
\hline 1 & 0 & 250 & - & - & 11,8 \\
\hline 2 & 0 & 230 & $1 / 100-n \quad 20$ & 0,00461 & 12,0 \\
\hline 3 & 0 & 200 & 50 & 0,0115 & 11,9 \\
\hline 4 & 0 & 150 & $\gg \quad 100$ & 0,023 & 11,9 \\
\hline 5 & 3 & 250 & - & - & 15,2 \\
\hline 6 & 3 & 245 & $1 / 100-n \quad 5$ & 0,00115 & 14,9 \\
\hline 7 & 3 & 230 & 20 & 0,00461 & 14,2 \\
\hline 8 & 3 & 200 & 50 & 0,0115 & 15,1 \\
\hline 9 & 3 & 150 & $\triangle 100$ & 0,023 & 14,8 \\
\hline 10 & 3 & 230 & $1 / 10-n \quad 20$ & 0,046 & 14,1 \\
\hline 11 & 3 & 200 & $\searrow$ & 0,1156 & 13,2 \\
\hline
\end{tabular}

Tabelle XVIII.

\begin{tabular}{|c|c|c|c|c|c|}
\hline $\begin{array}{l}\text { Versuchs- } \\
\text { nummer }\end{array}$ & $\begin{array}{l}\text { Dauer } \\
\text { der } \\
\text { Autolyse } \\
\text { Tage }\end{array}$ & $\begin{array}{l}\text { Destil- } \\
\text { liertes } \\
\text { Wasser } \\
\text { ccm }\end{array}$ & $\begin{array}{c}\text { Natrium- } \\
\text { chlorid- } \\
\text { lösung } \\
\text { ccm }\end{array}$ & $\begin{array}{l}\text { Na- } \\
\text { Gehalt } \\
\text { g }\end{array}$ & $\begin{array}{l}\text { Nicht koagulierter } N \\
\text { in } 50 \mathrm{ccm} \text { Filtrat } \\
\text { entsprechend } 1 / 10-n- \\
\text { Säure } \\
\text { in ccm }\end{array}$ \\
\hline 1 & 0 & 250 & - & - & 11,8 \\
\hline 2 & 0 & 230 & $1 / 10-n 10$ & 0,023050 & 12,2 \\
\hline 3 & 0 & 200 &,$\quad 50$ & 0,1152 & 12,5 \\
\hline 4 & 0 & 150 & D 100 & 0,2305 & 12,9 \\
\hline 5 & 3 & 250 & - & - & 18,2 \\
\hline 6 & 3 & 245 & $1 / 100-n \quad 5$ & 0,001152 & 17,5 \\
\hline 7 & 3 & 230 & $>\quad 20$ & 0,00461 & 17,5 \\
\hline 8 & 3 & 200 & $\triangle \quad 50$ & 0,01152 & 18,6 \\
\hline 9 & 3 & 150 & $>100$ & 0,023 & 16,3 \\
\hline 10 & 3 & 230 & $1 / 10-n \quad 20$ & 0,046 & 16,5 \\
\hline 11 & 3 & 200 & $\triangle 50$ & 0,1152 & 16,5 \\
\hline 12 & 3 & 180 & $>\quad 70$ & 0,1612 & 16,5 \\
\hline 13 & 3 & 150 & > 100 & 0,2305 & 15,5 \\
\hline
\end{tabular}


Das Kupferchlorid, das Chlornatrium und das Natriumsulfat verändern nicht, wenigstens nicht in merkbarer Weise, die Menge des $\mathrm{N}$ bei den vor der Autolyse verarbeiteten Proben; bei den Proben, wo die Autolyse stattgefunden hat, wird die Menge des nicht koagulierbaren Stickstoffs durch kleine Mengen der genannten Salze nicht beeinflußt, während größere Mengen dieser eine Abnahme der Stickstoffmenge bewirken, und zwar in der Weise, daß diese Abnahme parallel mit dem Ansteigen der zugesetzten Salzmenge schreitet.

8. Palladium-, Calcium-, Baryum-, Kalium-, Strontium-, Kadmium-, Nickel-, Magnesium-, Zinksalze.

Tabelle XIX.

\begin{tabular}{|c|c|c|c|c|c|}
\hline $\begin{array}{l}\text { Versuchs- } \\
\text { nummer }\end{array}$ & $\begin{array}{c}\text { Dauer } \\
\text { der } \\
\text { Autolyse } \\
\text { Tage }\end{array}$ & $\begin{array}{l}\text { Destil- } \\
\text { liertes } \\
\text { Wasser } \\
\text { ccm }\end{array}$ & \begin{tabular}{|} 
Palladium- \\
chlorür- \\
lösung \\
ccm
\end{tabular} & $\begin{array}{c}\text { Pd- } \\
\text { Gehalt } \\
\mathrm{g}\end{array}$ & $\begin{array}{l}\text { Nicht koagulierter } N \\
\text { in } 50 \text { ccm Filtrat } \\
\text { entsprechend } 1 / 10-n- \\
\text { Säure } \\
\text { in } \mathrm{ccm}\end{array}$ \\
\hline 1 & 0 & 250 & -- & - & 13,2 \\
\hline 2 & 0 & 249 & $1 / 100-n \quad 1$ & 0,0010 & 13,0 \\
\hline 3 & 0 & $24 \check{5}$ & $» \quad 5$ & 0,0050 & 12,8 \\
\hline 4 & 0 & 240 & $\gg \quad 10$ & 0,010 & 13,5 \\
\hline 5 & 0 & 200 & $\triangleright \quad 50$ & 0,050 & 13,3 \\
\hline 6 & 3 & 250 & - & - & 23,8 \\
\hline 7 & 3 & 249 & $1 / 100-n \quad 1$ & 0,0010 & 24,0 \\
\hline 8 & 3 & 247 & $\triangleright \quad 3$ & 0,0030 & 24,1 \\
\hline 9 & 3 & 245 & 5 & 0,0050 & 23,9 \\
\hline 10 & 3 & 240 & $» \quad 10$ & 0,010 & 23,7 \\
\hline 11 & 3 & 225 & $» \quad 25$ & 0,025 & 24,5 \\
\hline 12 & 3 & 250 & $\gg \quad 50$ & 0,050 & 24,4 \\
\hline
\end{tabular}


Tabelle XX.

\begin{tabular}{|c|c|c|c|c|c|}
\hline $\begin{array}{l}\text { Versuchs- } \\
\text { nummer }\end{array}$ & $\begin{array}{c}\text { Dauer } \\
\text { der } \\
\text { Autolyse } \\
\text { Tage }\end{array}$ & $\begin{array}{l}\text { Destil- } \\
\text { liertes } \\
\text { Wasser } \\
\text { ccm }\end{array}$ & $\begin{array}{c}\text { Calcium- } \\
\text { chlorid- } \\
\text { lösung } \\
\text { ccm }\end{array}$ & $\begin{array}{c}\text { Ca- } \\
\text { Gehalt } \\
\mathrm{g}\end{array}$ & $\begin{array}{l}\text { Nicht koagulierter N } \\
\text { in } 50 \text { ccm Filtrat } \\
\text { entsprechend } 1 / 10-n- \\
\text { Säure } \\
\text { in ccm }\end{array}$ \\
\hline 1 & 0 & 250 & - & - & 12,3 \\
\hline 2 & 0 & 230 & $1 / 100-n \quad 20$ & 0,00802 & 12,5 \\
\hline 3 & 0 & 200 & $\Rightarrow \quad 100$ & 0,0401 & 12,2 \\
\hline 4 & 3 & 250 & - & - & 17,2 \\
\hline 5 & 3 & 245 & $1 / 100-n$ & 0,0020 & 17,0 \\
\hline 6 & 3 & 240 & $\triangleright \quad 10$ & 0,0040 & 17,9 \\
\hline 7 & 3 & 230 & $\searrow \quad 20$ & 0,0080 & 17,9 \\
\hline 8 & 3 & 200 & . $\quad 50$ & 0,0200 & 18,0 \\
\hline 9 & 3 & 150 & $\gg \quad 100$ & 0,0401 & 17,9 \\
\hline
\end{tabular}

Tabelle XXI.

\begin{tabular}{|c|c|c|c|c|c|}
\hline $\begin{array}{l}\text { Versuchs- } \\
\text { nummer }\end{array}$ & $\begin{array}{c}\text { Dauer } \\
\text { der } \\
\text { Autolyse } \\
\text { Tage }\end{array}$ & $\begin{array}{l}\text { Destil- } \\
\text { liertes } \\
\text { Wasser } \\
\text { ccm }\end{array}$ & $\begin{array}{c}\text { Baryum- } \\
\text { chlorid- } \\
\text { lösung } \\
\text { ccm }\end{array}$ & $\begin{array}{c}\text { Ba- } \\
\text { Gehalt } \\
\text { g }\end{array}$ & $\begin{array}{l}\text { Nicht koagulierter } \mathrm{N} \\
\text { in } 50 \mathrm{ccm} \text { Filtrat } \\
\text { entsprechend } 1 / 10-\mathrm{n}- \\
\text { Säure } \\
\text { in } \mathrm{ccm}\end{array}$ \\
\hline 1 & 0 & 250 & - & - & 13,4 \\
\hline 2 & 0 & 200 & $1 / 100-n \quad 50$ & 0,0684 & 13,7 \\
\hline 3 & 0 & 150 & $\searrow \quad 100$ & 0,1374 & 13,9 \\
\hline 4 & 3 & 250 & - & - & 19,6 \\
\hline 5 & 3 & 249 & $1 / 100-n$ & 0,00137 & 20,3 \\
\hline 6 & 3 & 245 & $\gg \quad 5$ & 0,00685 & 20,3 \\
\hline 7 & 3 & 240 & > 10 & 0,0137 & 19,6 \\
\hline 8 & 3 & 225 & , 25 & 0,0342 & 19,1 \\
\hline 9 & 3 & 200 & $>\quad 50$ & 0,0684 & 19,5 \\
\hline 10 & 3 & 150 & $\gg \quad 100$ & 0,1374 & 19,3 \\
\hline
\end{tabular}


Tabelle XXII.

\begin{tabular}{|c|c|c|c|c|c|}
\hline $\begin{array}{l}\text { Versuchs- } \\
\text { nummer }\end{array}$ & $\begin{array}{c}\text { Dauer } \\
\text { der } \\
\text { Autolyse } \\
\text { Tage }\end{array}$ & $\begin{array}{l}\text { Destil- } \\
\text { liertes } \\
\text { Wasser } \\
\text { ccm }\end{array}$ & $\begin{array}{l}\text { Kalium- } \\
\text { bromid- } \\
\text { lösung } \\
\text { ccm }\end{array}$ & $\begin{array}{c}\text { K- } \\
\text { Gehalt } \\
\text { g }\end{array}$ & $\begin{array}{l}\text { Nicht koagulierter } \mathbb{N} \\
\text { in } 50 \mathrm{ccm} \text { Filtrat } \\
\text { entsprechend } 1 / 10-\mathrm{n}- \\
\text { Säure } \\
\text { in ccm }\end{array}$ \\
\hline 1 & 0 & 250 & - & - & 8,7 \\
\hline 2 & 0 & 225 & $1 / 100-n \quad 25$ & 0,00978 & 8,9 \\
\hline 3 & 0 & 150 & $\gg \quad 100$ & 0,03915 & 8,3 \\
\hline 4 & 3 & 250 & - & - & 12,0 \\
\hline 5 & 3 & 245 & $1 / 100-n \quad 5$ & 0,00195 & 12,0 \\
\hline 6 & 3 & 240 & $\gg \quad 10$ & 0,0038 & 12,2 \\
\hline 7 & 3 & 225 & 25 & 0,00978 & 11,9 \\
\hline 8 & 3 & 200 & $\gg \quad 50$ & 0,0195 & 12,0 \\
\hline 9 & 3 & 150 & $\gg \quad 100$ & 0,038 & 12,1 \\
\hline 10 & 3 & 225 & $1 / 10-n \quad 25$ & 0,0978 & 11,8 \\
\hline 11 & 3 & 200 & $\gg \quad 50$ & 0,195 & 11,9 \\
\hline 12 & 3 & 150 & $\gg \quad 100$ & 0,3915 & 11,7 \\
\hline
\end{tabular}

\begin{tabular}{|c|c|c|c|c|c|}
\hline $\begin{array}{l}\text { Versuchs- } \\
\text { nummer }\end{array}$ & $\begin{array}{l}\text { Dauer } \\
\text { der } \\
\text { Autolyse } \\
\text { Tage }\end{array}$ & $\begin{array}{l}\text { Destil- } \\
\text { liertes } \\
\text { Wasser } \\
\text { ccm }\end{array}$ & $\begin{array}{c}\text { Strontium. } \\
\text { bromid- } \\
\text { lösung } \\
\mathrm{ccm}\end{array}$ & $\begin{array}{l}\text { Sr- } \\
\text { Gehalt- } \\
\text { g }\end{array}$ & $\begin{array}{l}\text { Nicht koagulierter } N \\
\text { in } 50 \mathrm{ccm} \text { Filtrat } \\
\text { entsprechend } 1 / 10-\mathrm{n}- \\
\text { Säure } \\
\text { in ccm }\end{array}$ \\
\hline 1 & 0 & 250 & - & - & 7,5 \\
\hline 2 & 0 & 225 & $1 / 100-n \quad 25$ & 0,0219 & 7,2 \\
\hline 3 & 0 & 150 & $\begin{array}{l}> \\
100\end{array}$ & 0,0876 & 7,8 \\
\hline 4 & 3 & 250 & - & - & 12,4 \\
\hline 5 & 3 & 249 & $1 / 100-n$ & 0,0008 & 12,6 \\
\hline 6 & 3 & 240 & » 10 & 0,008 & 12,7 \\
\hline 7 & 3 & 225 & » 25 & 0,0219 & 12,2 \\
\hline 8 & 3 & 200 & 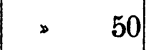 & 0,0438 & 11,6 \\
\hline 9 & 3 & 150 & » 100 & 0,0876 & 13,1 \\
\hline 10 & 3 & 225 & $1 / 10-n \quad 25$ & 0,219 & 12,9 \\
\hline 11 & 3 & 200 & $" \quad 50$ & 0,438 & 12,3 \\
\hline
\end{tabular}


Tabele XXIV.

\begin{tabular}{|c|c|c|c|c|c|}
\hline $\begin{array}{c}\text { Versuchs- } \\
\text { nummer }\end{array}$ & $\begin{array}{l}\text { Dauer } \\
\text { der } \\
\text { Autolysie } \\
\text { Tage }\end{array}$ & $\begin{array}{l}\text { Destil- } \\
\text { liertes } \\
\text { Wasser } \\
\text { ccm }\end{array}$ & \begin{tabular}{|} 
Stontium- \\
alorid- \\
ösung \\
ccm
\end{tabular} & $\begin{array}{l}\text { Sr- } \\
\text { Gehalt- } \\
\mathrm{g}\end{array}$ & $\begin{array}{l}\text { Nicht koagulierter } N \\
\text { in } 50 \mathrm{ccm} \text { Filtrat } \\
\text { entsprechend } 1 / 10-\mathrm{n}- \\
\text { Säure } \\
\text { in ccm }\end{array}$ \\
\hline 1 & 0 & 250 & - & - & 8,7 \\
\hline 2 & 0 & 200 & $1 / 30-n \quad 50$ & 0,040 & 8,9 \\
\hline 3 & 0 & 150 & . 100 & 0,080 & 8,2 \\
\hline 4 & 3 & 250 & - & - & 13,5 \\
\hline 5 & 3 & 240 & $1 / 10-n \quad 10$ & 0,008 & 13,9 \\
\hline 6 & 3 & 230 & 20 & 0,016 & 14,6 \\
\hline 7 & 3 & 220 & 30 & 0,024 & 14,1 \\
\hline 8 & 3 & 200 & 50 & 0,040 & 13,6 \\
\hline 9 & 3 & 150 & 100 & 0,08 & 13,5 \\
\hline 10 & 3 & 235 & $1 / 1-n \quad 15$ & 0,120 & 14,0 \\
\hline 11 & 3 & 225 & 25 & 0,200 & 13,2 \\
\hline
\end{tabular}

Tabele XXV.

\begin{tabular}{|c|c|c|c|c|c|}
\hline $\begin{array}{l}\text { Versuchs- } \\
\text { nummer }\end{array}$ & $\begin{array}{c}\text { Dauer } \\
\text { der } \\
\text { Autolyse } \\
\text { Tage }\end{array}$ & $\begin{array}{l}\text { Destil- } \\
\text { liertes } \\
\text { Wasser } \\
\text { ccm }\end{array}$ & \begin{tabular}{|} 
Cadmium- \\
alorid- \\
isung \\
ccm
\end{tabular} & $\begin{array}{l}\text { Gd- } \\
\text { Gehalt } \\
\text { g }\end{array}$ & $\begin{array}{c}\text { Nicht koagulierter } N \\
\text { in } 50 \text { ccm Filtrat } \\
\text { entsprechend } 1 / 10-n- \\
\text { Säure } \\
\text { in ccm }\end{array}$ \\
\hline 1 & 0 & 250 & - & - & 9,4 \\
\hline 2 & 0 & 230 & $1 / p_{0}-\mathrm{n} 20$ & 0,02248 & 9,0 \\
\hline 3 & 0 & 200 & $\gg \quad 50$ & 0,0561 & 8,7 \\
\hline 4 & 3 & 250 & - & - & 14,9 \\
\hline 5 & 3 & 249 & tpo-n 1 & 0,001124 & 14,1 \\
\hline 6 & 3 & 245 & $» \quad 5$ & 0,00561 & 15,6 \\
\hline 7 & 3 & 240 & × 10 & 0,01124 & 14,2 \\
\hline 8 & 3 & 230 & * $\quad 20$ & 0,02248 & 12,5 \\
\hline 9 & 3 & 220 & - 30 & 0,03372 & 12,3 \\
\hline 10 & 3 & 200 & " 50 & 0,0561 & 11,9 \\
\hline 11 & 3 & 150 & * 100 & 0,1124 & 11,8 \\
\hline
\end{tabular}


Tabelle XXVI.

\begin{tabular}{|c|c|c|c|c|c|}
\hline $\begin{array}{l}\text { Versuchs- } \\
\text { nummer }\end{array}$ & $\begin{array}{l}\text { Dauer } \\
\text { der } \\
\text { Autolyse } \\
\text { Tage }\end{array}$ & $\begin{array}{l}\text { Destil- } \\
\text { liertes } \\
\text { Wasser } \\
\text { ccm }\end{array}$ & $\begin{array}{l}\text { Nickel- } \\
\text { chlorür- } \\
\text { lösung } \\
\text { ccm }\end{array}$ & $\begin{array}{l}\mathrm{Ni}- \\
\text { Gehalt } \\
\mathrm{g}\end{array}$ & $\begin{array}{l}\text { Nicht koagulierter: } N \\
\text { in } 50 \mathrm{ccm} \text { Filtratt } \\
\text { entsprechend } 1 / 10-\mathrm{-n}- \\
\text { Säure } \\
\text { in ccm }\end{array}$ \\
\hline 1 & 0 & 250 & - & - & 11,8 \\
\hline 2 & 0 & 200 & $1 / 100-n \quad 50$ & 0,02935 & 11,5 \\
\hline 3 & 0 & 150 & . 100 & 0,0587 & 10,9 \\
\hline 4 & 3 & 250 & - & - & 13,5 \\
\hline 5 & 3 & 249 & $1 / 100-n-1$ & 0,000587 & 13,5 \\
\hline 6 & 3 & 240 & , 10 & 0,00587 & 13,6 \\
\hline 7 & 3 & 230 & $\triangleright \quad 20$ & 0,01074 & 13,2 \\
\hline 8 & 3 & 200 & $\triangle \quad 50$ & 0,02935 & 11,9 \\
\hline 9 & 3 & 150 & . 100 & 0,0587 & 11,9 \\
\hline
\end{tabular}

Tabelle XXVII.

\begin{tabular}{|c|c|c|c|c|c|}
\hline $\begin{array}{l}\text { Versuchs- } \\
\text { nummer }\end{array}$ & $\begin{array}{l}\text { Dauer } \\
\text { der } \\
\text { Autolyse } \\
\text { Tage }\end{array}$ & $\begin{array}{c}\text { Destil- } \\
\text { liertes } \\
\text { Wasser } \\
\text { ccm }\end{array}$ & $\begin{array}{l}\text { Nickel- } \\
\text { nitrat- } \\
\text { lösung } \\
\text { ccm }\end{array}$ & $\begin{array}{c}\text { Ni- } \\
\text { Gehalt } \\
\text { g }\end{array}$ & $\begin{array}{c}\text { Nicht koagulierter N } \\
\text { in } 50 \mathrm{ccm} \text { Filtratt } \\
\text { entsprechend } 1 / 10-; n- \\
\text { Säure } \\
\text { in ccm }\end{array}$ \\
\hline 1 & 0 & 250 & - & - & 11,8 \\
\hline 2 & 0 & 200 & $1 / 100-n \quad 50$ & 0,02935 & 11,5 \\
\hline 3 & 0 & 150 & $\triangle 100$ & 0,0587 & 10,0 \\
\hline 4 & 3 & 250 & - & - & 18,5 \\
\hline$\check{5}$ & 3 & 249 & $1 / 100-n \quad 1$ & 0,000587 & 18,9 \\
\hline 6 & 3 & 240 & $\Rightarrow \quad 10$ & 0,00587 & 18,5 \\
\hline 7 & 3 & 230 & \20 & 0,01074 & 18,3 \\
\hline 8 & 3 & 200 & $\triangle \quad 50$ & 0,02955 & 16,9 \\
\hline 9 & 3 & 150 & . 100 & 0,0587 & 15,1 \\
\hline
\end{tabular}


Tabelle XXVIII.

\begin{tabular}{|c|c|c|c|c|c|}
\hline $\begin{array}{l}\text { Versuchs- } \\
\text { nummer }\end{array}$ & $\begin{array}{c}\text { Dauer } \\
\text { der } \\
\text { Autolyse } \\
\text { Tage }\end{array}$ & $\begin{array}{c}\text { Destil- } \\
\text { liertes } \\
\text { Wasser } \\
\text { ccm }\end{array}$ & $\begin{array}{l}\text { Magne- } \\
\text { sium- } \\
\text { chlorid- } \\
\text { lösung } \\
\text { ccm }\end{array}$ & $\begin{array}{c}\text { Mg- } \\
\text { Gehalt } \\
\text { g }\end{array}$ & $\begin{array}{l}\text { Nicht koagulierter } N \\
\text { in } 50 \mathrm{ccm} \text { Filtrat } \\
\text { entsprechend } 1 / 10-\mathrm{n}- \\
\text { Säure } \\
\text { in ccm }\end{array}$ \\
\hline 1 & 0 & 250 & - & - & 7,3 \\
\hline 2 & 0 & 200 & $1 / 100-n \quad 50$ & 0,01218 & 6,5 \\
\hline 3 & 0 & 150 & $\Rightarrow \quad 100$ & 0,02436 & 6,0 \\
\hline 4 & 3 & 250 & - & - & 14,9 \\
\hline 5 & 3 & 245 & $1 / 100-n \quad 5$ & 0,001218 & 14,8 \\
\hline 6 & 3 & 230 & $\begin{array}{l}>\quad 20 \\
\end{array}$ & 0,00486 & 14,3 \\
\hline 7 & 3 & 200 & $\begin{array}{l}>\quad 50 \\
\end{array}$ & 0,01218 & 13,1 \\
\hline 8 & 3 & 150 & > 100 & 0,0236 & 13,1 \\
\hline
\end{tabular}

Tabelle XXIX.

\begin{tabular}{|c|c|c|c|c|c|}
\hline $\begin{array}{l}\text { Versuchs- } \\
\text { nummer }\end{array}$ & $\begin{array}{l}\text { Dauer } \\
\text { der } \\
\text { Autolyse } \\
\text { Tage }\end{array}$ & $\begin{array}{l}\text { Destil- } \\
\text { liertes } \\
\text { Wasser } \\
\text { ccm }\end{array}$ & $\begin{array}{l}\text { Zink- } \\
\text { sulfat- } \\
\text { lösung } \\
\text { ccm }\end{array}$ & $\begin{array}{c}\text { Zn- } \\
\text { Gehalt } \\
\text { g }\end{array}$ & $\begin{array}{l}\text { Nicht koagulierter } N \\
\text { in } 50 \text { ccm Filtrat } \\
\text { entsprechend } 1 / 10-n-n \\
\text { Saure } \\
\text { in ccm }\end{array}$ \\
\hline 1 & 0 & 250 & - & - & 8,3 \\
\hline 2 & 0 & 225 & $1 / 100-n 25$ & 0,01635 & 7,7 \\
\hline 3 & 0 & 200 & $>\quad 50$ & 0,0327 & 7,9 \\
\hline 4 & 0 & 150 & , 100 & 0,0654 & 7,0 \\
\hline 5 & 3 & 250 & - & - & 12,6 \\
\hline 6 & 3 & 245 & $1 / 100-n \quad 5$ & 0,00327 & 12,9 \\
\hline 7. & 3 & 240 & $>\quad 10$ & 0,00654 & 12,0 \\
\hline 8 & 3 & 225 & $\Rightarrow \quad 25$ & 0,01635 & 11,4 \\
\hline 9 & 3 & 200 & × 50 & 0,0327 & 10,5 \\
\hline 10 & 3 & 150 & , 100 & 0,0654 & 9,9 \\
\hline
\end{tabular}

Hoppe-Seyler's Zeitschrift f. physiol. Chemie. LX. 
Aus den Versuchsprotokollen geht hervor, daß die Salze dieser Metalle den autolytischen Prozeß nicht beeinflussen; der Zusatz einiger derselben $z u$ dem Leberbrei hat nämlich zu Ende der Autolyse keine merkbaren Änderungen der Menge des nicht koagulierbaren $\mathrm{N}$ bewirkt, oder, wenn eine Änderung eintrat, erfolgte diese in derselben Richtung und unter dem Einfluß derselben Salzmenge, welche dieselbe Änderung der unkoagulierbaren $\mathrm{N}$-Menge vor der Autolyse bewirkt hatte.

Hier sind einige Betrachtungen am Platze über den Mechanismus, auf welchem die Zunahme des nicht koagulierbaren Stickstoffs in den Fällen zurückzuführen ist, in welchen sie infolge des Zusatzes eines Metallsalzes stattfindet. Man könnte einwenden, daß durch die Salze nicht die Autolyse, sondern die Eiweißstoffe beeinflußt werden; denn wir sahen, daß dieselben Salze die Menge des unkoagulierbaren N, auch bevor die Autolyse stattgefunden hat, erhöhen, sofern nur der Zusatz größer ist; man könnte somit annehmen, daß geringere Salzmengen eine entsprechend stärkere Wirkung entfalten, sobald sie eine längere Zeitperiode, nämlich diejenige, welche für die Autolyse notwendig ist, einwirken können.

Es scheint uns jedoch, daß die eben erwähnte Annahme bei einer näheren Untersuchung und Erwägung unbegründet erscheint. Die letzte Gruppe von Versuchen beweist nämlich, daß diejenigen Salze, welche die Autolyse nicht beeinflussen, dieselbe Wirkung auf die nicht koagulierbare N-Menge ausüben, sowohl wenn die Koagulation sofort als auch wenn sie erst nach vollendeter Autolyse stattfindet.

Folglich könnte die angenommene langsame Wirkung der zugesetzten und aktiv erschienenen Salze nur katalytischer Natur sein. Von diesem Standpunkte ist die Frage jedenfalls schwerer zu lösen; anderseits ist aber ihre Lösung von geringerer Bedeutung.

Wir haben nichtsdestoweniger versucht, ihrer Entscheidung soweit als möglich näher zu treten, und zu diesem Zwecke untersucht, ob die Metalle, deren Salze sich aktiv erwiesen hatten, imstande waren, während einer gewissen Zeitperiode, unabhängig von der Autolyse, ähnliche Veränderungen der Eiweißstoffe zu bewirken. 
So haben wir die Wirkung nntersucht, welche die genannten Salze auf gekochten Leberbrei ausüben und die Menge des unkoagulierbaren $\mathrm{N}$ sowohl unmittelbar nach dem Salzzusatze als in verschiedenen Zeitabständen bestimmt; allerdings handelt es sich hier um denaturierte Eiweißkörper.

Tabelle XXX.

\begin{tabular}{|c|c|c|c|c|}
\hline $\begin{array}{c}\text { Leber- } \\
\text { brei }\end{array}$ & $\begin{array}{l}\mathrm{H}_{2} \mathrm{O} \\
\mathrm{ccm}\end{array}$ & $\begin{array}{c}\text { Zusatz } \\
\text { (nach dem Kochen) } \\
\text { ccm }\end{array}$ & $\begin{array}{l}\text { Nicht koagulie } \\
\text { Filtrat) entspr } \\
\text { sofort nach } \\
\text { dem Kochen } \\
\text { ccm }\end{array}$ & $\begin{array}{c}\mathrm{N} \text { (in } 50 \mathrm{ccm} \\
\text { end }{ }^{1} \mid 10-\mathrm{n}-\mathrm{S} \text { äure } \\
\text { nach weiteren } \\
3 \text { Tagen } \\
\text { Thermostaten } \\
\text { bei } 37^{\circ} \\
\text { ccm }\end{array}$ \\
\hline 20 & 250 & - & 10,0 & 12,5 \\
\hline 20 & 230 & Eisenchlorür $1 / 100-n 20$ & 10,2 & 11,6 \\
\hline 20 & 200 & $\triangleright \quad 50$ & 10,2 & 11,1 \\
\hline 20 & 230 & Mangansulfat $\gg 20$ & 10,4 & 11,7 \\
\hline 20 & 200 & $\triangle \quad \triangleright \quad 50$ & 10,0 & 12,0 \\
\hline
\end{tabular}

Kontrolle bei gewöhnlicher Autolyse $=16,9 \mathrm{ccm} \mathrm{1/10-n-Säure.}$

Anmerkung: Der gewöhnliche Zusatz von 1\% Monokaliumphosphat erfolgte natürlich erst zu Ende des Versuches, zum Zwecke der vollständigen Enteiweißung.

In folgender Versuchsreihe 31 (Tab. XXXI), bei welcher der gleiche Leberbrei wie in Tabelle XXX zur Verwendung kam, erfolgte der Salzzusatz, bevor die Leberbreiaufschwemmung gekocht wurde.

Die Resultate weichen nicht von den in Versuchsreihe $\mathbf{3 0}$ enthaltenen $\mathbf{a b}$.

Kleine Eisensalzmengen beeinflussen demnach gekochte Leberbreiaufschwemmungen auch bei längerer Einwirkung in keiner Weise, ebensowenig wie ihr Zusatz vor dem Kochen.

Das Gesagte können wir nun folgendermaßen kurz zusammenfassen:

1. Eisenchlorid, Sulfat, Oxalat, Manganchlorid, -Sulfat, -Acetat und -Lactat, Platinchlorid, Aluminium- 
Tabelle XXXI.

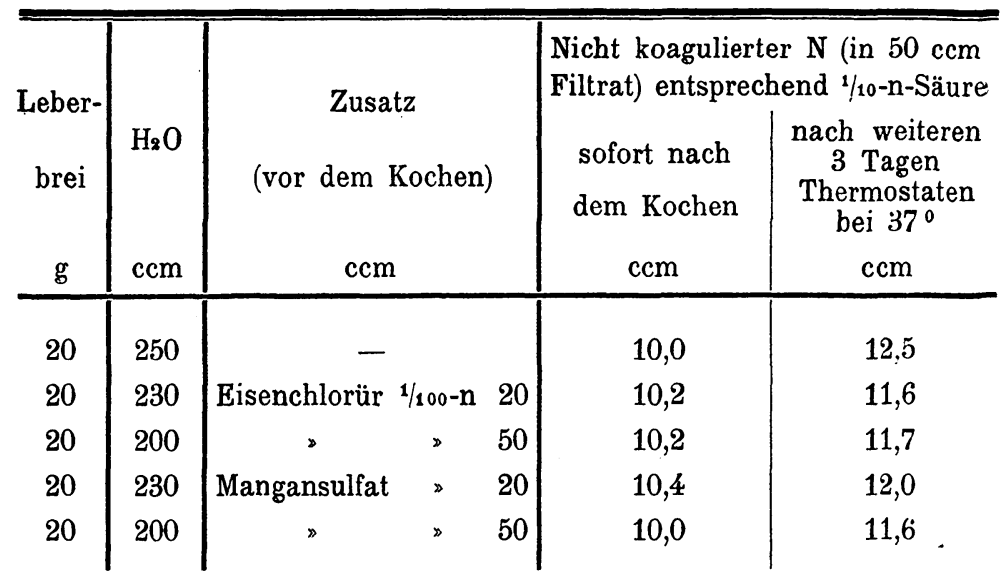

chlorid und -Sulfat, Kobaltchlorid und-Nitrat wirken folgendermaßen: wenn sie dem Leberbrei in kleinen Mengen zugesetzt werden - und zwar so klein, da $B$ diese Mengen an und für sich die Eiweißstoffe nicht derartig beeinflussen, daß sie eine quantitative Änderung der unkoagulierbaren N-Menge hervorrufen-bewirken sie, bei stattgefundener Autolyse, eine Vermehrung des nicht koagulierbaren Stickstoffs. Diese Vermehrung ist eine progressive und nimmt $z u$ mit dem Zunehmen der zugesetzten Salzmenge.

Eine Abweichung vondieser Wirkungsweise zeigen folgendeSalze:Manganacetat, KobaltchloridundPlatinchlorid. Die ersten zwei bewirken eine Vermehrung des unkoagulierbaren Stickstoffs, bis die Menge des zugesetzten Salzes eine gewisse Höhe erreicht hat; wenn diese Grenze überschritten wird, tritt an Stelle der Vermehrung eine Verminderung des unkoagulablen $\mathrm{N}$ ein.

Platinchlorid bewirkt mit dem Steigen der Menge, die zugesetzt wird, abwechselnd eine Vermehrung und eine Verminderung des nicht koagulablen Stickstoffs.

2. Chlornatrium, Natriumsulfat und Kupfersulfat üben, wenn sie in kleinen Mengen zugesetzt werden, 
keinen merkbaren quantitativen Einfluß auf die Menge des koagulierbaren $\mathrm{N}$ aus; wenn sie in größeren Mengen zugesetzt werden (diese dürfen jedoch nicht so groß sein, daß sich bei den Versuchen mit Koagulation vor der Autolyse Unterschiede zwischen den Proben mit und denjenigen ohne Salze herausstellen), führen sie dagegen eine Verminderung des unkoagulablen Stickstoffes herbei.

3.Palladiumchlorid, Chlorcalcium, Baryumchlorid, Strontiumchlorid und -Bromid beeinflussen nicht in merkbarer Weise dieStickstoffmenge, sodaß kein sichtbarer Unterschied $z$ wischen den Proben mit und denjenigen ohne Zusatz der Salze vorhanden ist.

Cadmiumchlorid, Nickelchlorid und-Nitrat, Magnesiumchlorid und Zinksulfat bewirken eine Verminderung des nicht koagulierbaren $\mathrm{N}$; dieselbe ist aber schon, bevor der Leberbrei der Autolyse überlassen wird, nachweisbar.

Die Inaktivität der sub 3 genannten Salze und die Ähnlichkeit zwischen der Wirkung der verschiedenn Salze ein und desselben Metalles beweisen, daß die auf die Autolyse ausgeübte Wirkung größtenteils dem Kation zuzuschreiben ist, während dem Anion höchstens eine Nebenwirkung zukommt, welche sich darauf beschränkt, die Hauptwirkung in verschiedener Weise zu modifizieren.

Es reiht sich die auseinandergesetzte Wirkung der Salze auf die Autolyse den mannigfachen anderen (Ausflockung von Kolloiden, Agglutination von Blutkörperchen und Bakterien usw.) an, denen sich in den letzten Jahren die Forschung mit wachsendem Interesse zugewendet hat.

Wenn auch in den Hauptlinien eine grobe Übereinstimmung mit der Beeinflussung dieser Vorgänge angedeutet zu sein scheint (hauptsächlich in den Beziehungen zur Wertigkeit und Entladungsspannung der Kationen), so ist jedoch ein näherer Vergleich nicht statthaft. In den niedergelegten Versuchsreihen beschränkten wir uns nämlich darauf, den Einfluß verschiedener 
Verbindungen auf die Gesamtautolyse zu prüfen: wir könnten also die Metalle höchstens nach dem Grade, in welchem sie dieselbe befördern, ordnen. Dies wird aber durch den Umstand verboten, daß die Autolyse verschiedener Leberbreie auch durch gleiche Mengen einer und derselben Substanz in verschiedenem Maße beeinflußt wird, entsprechend ihrem Verhalten bei der einfachen Autolyse, ohne jeglichen Zusatz, und wir mußten natürlich in jeder Versuchsreihe einen verschiedenen Leberbrei heranziehen.

Stellen wir unsere Resultate den kürzlich von Izar ${ }^{1}$ ) im hiesigen Institute bei dem Studium des Einflusses von Metallhydrosolen auf die Autolyse gegenüber, so ergibt sich, mit Ausnahme des $\mathrm{Pd}$, eine befriedigende Übereinstimmung, indem dieselben Metalle auch im Kolloidzustande diesen Prozeß aktivieren.

1) Biochem. Zeitschrift, 1909. 